\title{
From evidence-based medicine to evidence-informed practice: Comments on Turner et al. 2020
}

To the Editor

Psychiatry is a unique healthcare area where the bridge between the biomedical and social sciences is a central focus of care delivery. Complex psycho-socio-cultural factors impact the aetiology and outcomes of mental disorders and of related concerns such as suicidality. In their recent article, Turner et al. (2020) clearly articulate the value of combining the Zero Suicide Framework with principles of a Just Restorative Culture. This article also acknowledges ongoing debates and concerns about the Zero Suicide Movement. Castle and Goldney (2018) highlight concerns about its inaccurate use of the term 'evidence-based', the clinical burden associated with the expectation to reach an aspirational target and the potential to deepen feelings of guilt for all those involved with the suicide. With successful implementation, the cultural change such as described by Turner et al. works to address these concerns.

However, there is a risk that over-reliance on the principles of a framework can lead to a processheavy approach. Rigid application of research-based evidence and guidelines to complex and multifactorial human experiences may fail to acknowledge the reality that variance in adverse outcomes (i.e. same intervention, different outcome) will persist regardless of the effectiveness of any intervention. Regardless of this, a strength of Turner et al.'s approach is the acknowledgement of the human impact of these events on the multiple people affected by the event. We think that it is vital that in the focus of process that opportunities for the profession and organisations to learn from these events are not lost. Innovative approaches to understand and analyse adverse clinical outcomes, especially when processes were adequately followed, is urgently needed in psychiatry. Understanding these variances also requires organisations that can provide the time, space and support for reflective practice - an opportunity that may not be readily available in contemporary mental health services (Looi and Maguire, 2019). Creating these opportunities will enhance a move from evidence-based medicine focused on the process to evidence-informed practice focused on each patient within the process. 


\section{References}

1. Castle, DJ, Goldney, RD (2018) Is a zero suicide target defensible and sensible? Australasian Psychiatry 26: 671.

2. Looi, JC, Maguire, $P$ (2019) Surviving the Heart of Darkness: Reflections on the experience of psychiatrists working in public mental health services in Australia. Australasian Psychiatry 27: 634-636.

3. Turner, K, Stapelberg, NJ, Sveticic, J, et al. (2020) Inconvenient truths in suicide prevention: Why a Restorative Just Culture should be implemented alongside a Zero Suicide Framework. Australian and New Zealand Journal of Psychiatry 54: 571-581. 